\title{
Doxastische Selbstkontrolle und Wahrheitssensitivität: Descartes und Spinoza über die Voraussetzungen einer rationalistischen Ethik der Überzeugungen
}

\author{
Renz, Ursula
}

\begin{abstract}
This paper discusses Descartes' and Spinoza's epistemological approaches under the perspective of how they contribute to the project of an ethics of belief. I argue that the problem of doxastic voluntarism is only one of two issues to be addressed within an ethics of belief. Another equally important concern is the question of how we can make sure that the rational control over our judgments does not override the truth-sensitivity of our beliefs. It is against this background that I compare Descartes' and Spinoza's views on how we may have some rational control over our own beliefs
\end{abstract}

DOI: https://doi.org/10.1515/agph-2014-0020

Posted at the Zurich Open Repository and Archive, University of Zurich ZORA URL: https://doi.org/10.5167/uzh-136674

Journal Article

Published Version

Originally published at:

Renz, Ursula (2014). Doxastische Selbstkontrolle und Wahrheitssensitivität: Descartes und Spinoza über die Voraussetzungen einer rationalistischen Ethik der Überzeugungen. Archiv für Geschichte der Philosophie, 96(4):463-488.

DOI: https://doi.org/10.1515/agph-2014-0020 
Ursula Renz

\title{
Doxastische Selbstkontrolle und Wahrheitssensitivität: Descartes und Spinoza über die Voraussetzungen einer rationalistischen Ethik der Überzeugungen
}

\begin{abstract}
This paper discusses Descartes' and Spinoza's epistemological approaches under the perspective of how they contribute to the project of an ethics of belief. I argue that the problem of doxastic voluntarism is only one of two issues to be addressed within an ethics of belief. Another equally important concern is the question of how we can make sure that the rational control over our judgments does not override the truth-sensitivity of our beliefs. It is against this background that I compare Descartes' and Spinoza's views on how we may have some rational control over our own beliefs.
\end{abstract}

Ursula Renz: Institut für Philosophie, Universität Klagenfurt, Universitätsstrasse 65-67, 9020 Klagenfurt; Ursula.Renz@aau.at

\section{Einführung}

Es gehört zu den bemerkenswertesten Tendenzen vieler erkenntnistheoretischer Ansätze der frühen Neuzeit, dass sie das Haben von gewissen Ideen zu einem Gegenstand von implizit normativen Erwägungen machen. In der Literatur ist in Anlehnung an William Kindon Clifford und an William James auch von einer „Ethics of Belief“ die Rede. ${ }^{1}$ Anders als in der Kontroverse zwischen James und Clifford liegt allerdings der Fokus in der erkenntnistheoretischen Diskussion der frühen Neuzeit nicht bei der Rechtfertigung religiösen Glaubens, sondern in der Klärung der Bedingungen der Möglichkeit einer rationalen Steuerung der Aneignung von Überzeugungen und Erkenntnissen überhaupt. Anvisiert wird also ein allgemeines erkenntnistheoretisches Programm, weswegen ich in der Folge von einer Ethik der Überzeugungen, und nicht des Glaubens sprechen möchte.

1 James 1956 (erstmals 1896). Bernard Williams hat die Frage des epistemischen Voluntarismus 1973 wieder aufgegriffen. Curley 1975 hat den Terminus „ethics of belief“ in die Diskussion über die Erkenntnistheorie der frühen Neuzeit eingespeist. 
Wie plausibel ist es, das Haben und Erwerben von Überzeugungen normativen Forderungen zu unterwerfen?

Prima facie scheint das Projekt einer Ethik der Überzeugungen einen schwierigen Stand $\mathrm{zu}$ haben. Zwar wird heute oft angenommen, dass „Wissen“ und „Erkenntnis“, aber auch „Überzeugung“ normative Prädikate sind. Die Rede ist auch von einem „value turn“ in der Erkenntnistheorie. ${ }^{2}$ Doch inwiefern sich daraus epistemische Pflichten und Lizenzen ableiten lassen, ist nicht klar. Auf der andern Seite können durchaus gewisse regulative Prinzipien identifiziert werden, die beim Erwerb von Wissen beachtet werden sollten. Ferner wird anerkannt, dass es so etwas wie epistemische Tugenden gibt, die sich günstig auf die epistemische Bilanz auswirken. ${ }^{3}$ Aber ob - und wenn ja: wie genau - die behaupteten regulativen Prinzipien oder epistemischen Tugenden mit derjenigen Normativität zusammenhängen, die für das Erkenntnisprädikat konstitutiv ist, lässt sich auch nach einigen Jahrzehnten analytischer Erkenntnistheorie schwer sagen.

Im Vergleich zu dieser Diskussion sind die Ansätze zu einer Ethik der Überzeugungen, wie man sie im Umkreis des klassischen Rationalismus findet, deutlich ambitionierter. Denn obwohl der Methodentraktat in der frühen Neuzeit zu einer bevorzugten philosophischen Textsorte wird, beschränken sich die meisten Philosophen nicht darauf, etwaige instrumentelle methodische Vorkehrungen zu spezifizieren, die wir im Vorlauf des Erkenntnisprozesses treffen sollten. Es geht aber auch nicht einfach darum, epistemische Dispositionen zu definieren, die für das Erkennen günstig sind. ${ }^{4}$ Im Fokus sind, mit anderen Worten, nicht primär die Rahmenbedingungen von Erkenntnis- bzw. Überzeugungsbildungsprozessen, sondern die zu bildenden Überzeugungen selber. Zur Diskussion steht, welche Überzeugungen man haben und welche man dagegen besser vermeiden sollte, wenn man nach Erkenntnis strebt.

Sieht man einmal davon ab, dass ein solches Programm skeptizistischen Einwände ausgesetzt ist - wird hier überhaupt ein erreichbares Ziel anvisiert? -, stellt sich die Frage, unter welchen Bedingungen eine Ethik der Überzeugungen ein sinnvolles Unterfangen ist. Vor allem zwei Voraussetzungen sind zu bedenken. Zum einen ist zu klären, inwiefern wir einen Einfluss darauf nehmen können, welche Überzeugungen wir haben. Dieses Problem wird oft unter dem Stichwort des doxastischen Voluntarismus abgehandelt, doch eigentlich ist es weiter zu fassen, nämlich als Frage nach der Möglichkeit einer rationalen Kontrolle der eigenen Überzeugungen. Zur Diskussion steht nicht einfach, ob wir uns

2 Siehe Kim 1988 für eine mittlerweile klassische Analyse sowie Pritchard 2007 für einen Überblick.

3 Siehe Greco/Turri 2011 für einen Überblick.

4 Siehe dazu auch Williams 1981, $132 \mathrm{f}$. 
willentlich dazu entscheiden können, von etwas überzeugt zu sein, sondern es geht grundsätzlicher darum, ob unsere geistigen Fähigkeiten von der Art sind, dass wir es „in der Hand haben“, gezielt Erkenntnis zu gewinnen. Es dürfte klar sein: Wie viel Kredit man einer Ethik der Überzeugungen einräumt, hängt maßgeblich von der genauen Antwort ab, die man auf diese Frage gibt. ${ }^{5}$

Es gibt indessen noch eine weitere Voraussetzung, die in diesem Zusammenhang erörtert werden muss, die jedoch in der Diskussion über die Ethik der Überzeugungen seltener thematisiert wird. Setzen wir einmal voraus, wir können tatsächlich einen Einfluss auf die Prozesse der eigenen Überzeugungsbildung ausüben und somit direkt oder indirekt steuern, welche Überzeugungen wir haben. Wie können wir dann sicher sein, dass unsere Einflussnahme auf Überzeugungen nicht einfach auf den Verlust der für Erkenntnis konstitutiven Geist-auf-Welt-Ausrichtung hinausläuft und unsere Überzeugungen sich nicht unter der Hand unseren Wünschen und Zielen statt „der Welt“ anpassen? Oder anders gefragt: Wie kann man sicher sein, dass die doxastische Selbstkontrolle so erfolgt, dass die Wahrheitssensitivität unserer Überzeugungen erhalten bleibt und die Bildung von Überzeugungen nicht „hinter unserem Rücken“ von unserem Wunschdenken gesteuert wird? ${ }^{6}$

Insgesamt lässt sich somit die Aufgabe, die eine Ethik der Überzeugungen vorgängig lösen muss, auf folgenden Nenner bringen: Es gilt zu klären, wie ein rationaler Umgang mit den eigenen Überzeugungen aussehen könnte, der auf der einen Seite die Möglichkeit einräumt, dass wir auf unsere eigenen Überzeugungen Einfluss nehmen können, der aber zugleich sicher stellt, dass dadurch die Wahrheitssensitivität unseres Denkens nicht geschmälert wird.

Blickt man vor diesem Hintergrund auf die Erkenntnistheorie der frühen Neuzeit, so wird man feststellen, dass die Suche nach einer Ethik der Überzeugungen zwar ein von vielen Philosophen geteilter Problemhorizont ist, die verschiedenen Autoren in ihrer Herangehensweise aber erheblich voneinander abweichen. ${ }^{7}$ Nicht alle Autoren gehen davon aus, dass die Möglichkeit, auf die

5 Siehe Vitz 2010 für eine allgemeine Darstellung des epistemischen Voluntarismus sowie Alston 1988 für verschiedene Möglichkeiten der Konzeption willentlicher Einflussnahme.

6 Dadurch würde nicht nur die Möglichkeit von Erkenntnis, sondern auch die Praxis der Zuschreibung von Überzeugungen unterminiert, besteht doch, wie etwa Haslanger 2001, 470 f., deutlich macht, ein konstitutiver Zusammenhang zwischen dem Haben von Überzeugungen und dem Streben nach Wahrheit. Es gehört zum Wesen des Überzeugt-Seins, dass das Subjekt annimmt, es sei im Besitz wahrer Auffassungen.

7 Einer der anonymen Gutachter hat die kritische Rückfrage aufgeworfen, ob diese Einschätzung mit Blick auf die Empiristen wirklich stimme. Meines Erachtens kann diese Frage weder einfach bejaht, noch verneint werden. Sicherlich gehen weder John Locke, noch David Hume davon aus, dass wir über das Haben einfacher Ideen eine willentliche Kontrolle ausüben kön- 
eigenen Überzeugungen einen Einfluss nehmen zu können, davon abhängt, dass wir uns willentlich zu bestimmten Überzeugungen entschließen können. Nicht alle Autoren sehen also in einem doxastischen Voluntarismus eine notwendige Voraussetzung für die Möglichkeit rationaler doxastischer Selbstkontrolle. Und nicht weniger groß fallen die Unterschiede im Blick darauf aus, wie die Wahrheitssensitivität doxastischer Selbstkontrolle sichergestellt wird. Diese Differenzen im Detail aufzuweisen, kann nicht die Aufgabe eines Aufsatzes sein. Ich möchte mich daher in der Folge darauf beschränken, exemplarisch anhand von Descartes und Spinoza zu zeigen, wie unterschiedlich die Ansätze sind, die allein schon innerhalb des Rationalismus zur Verfügung stehen.

Den Auftakt meiner Darlegungen wird eine Erörterung von Descartes' Antworten auf die Einwände von Hobbes und Mersenne zu seinen Meditationen bilden. Wie ich zeigen werde, nimmt Descartes in seiner Erkenntnistheorie nicht allein den Willen, sondern auch den Verstand in die Pflicht. Es ist der Verstand, der aus der Einsicht in die Dunkelheit einer bestimmten Idee den Schluss ziehen muss, dass sich der Wille der Zustimmung zu ihr enthalten soll. Obwohl die Aktivität des Urteilens Sache des Willens ist, gehört es zur Verantwortung des Verstandes, Ideen auf ihre Richtigkeit und Glaubwürdigkeit hin zu überprüfen. Insgesamt geht Descartes' Ansatz einer Ethik der Überzeugung von einer Art Gewaltenteilung aus: Das Urteil wird zwar vom Willen vollzogen, doch um zu sehen, ob es vollzogen werden darf, bedarf es des Verstandes.

Vor diesem Hintergrund werde ich mich im zweiten Teil Spinoza zuwenden und fragen, inwiefern er in seiner Ethik dem Subjekt eine Kontrolle über seine eigenen Überzeugungen zugesteht. Das scheint prima facie keine Option zu sein, denn bekanntlich ist Spinoza ein radikaler Kritiker von Descartes' doxastischem Voluntarismus. Überhaupt kritisiert Spinoza zahlreiche begriffliche Voraussetzungen des kartesischen Ansatzes. Es gibt allerdings einen Punkt, in dem Spinoza mit Descartes einig ist: Auch Spinoza lässt sich von der Intuition leiten, dass es für den epistemischen Wert einer Überzeugung einen Unterschied macht, ob wir plausible Gründe für sie haben oder ob sie sich nur externen psychologischen Einflüssen verdankt. Wie ich zeigen werde, lässt sich vor diesem Hintergrund auch

nen. Das heisst aber nicht, dass wir auf unsere Überzeugungen, die ja keineswegs mit den einfachen Ideen der Empiristen gleichzusetzen sind, keinerlei Einfluss nehmen können. Sicherlich sind ferner beide, was die Wahrheitssensitivität von Überzeugungen betrifft, skeptischer als die Rationalisten. Aber damit ist keineswegs jegliches Interesse an einer Ethik der Überzeugungen vom Tisch, sondern es verändert sich dahingehend, dass nunmehr die Frage in den Vordergrund rückt, wie man sich ohne die Garantien, welche die Rationalisten angenommen haben, in seinen Überzeugungen orientieren kann, vgl. dazu etwa Wolterstorffs Interpretation von Locke in Wolterstorf 1996, $11 \mathrm{f}$. 
die Unterscheidung zwischen dem Begriff der Handlung und dem des Leidens, wie er in Spinozas Affektenlehre scheinbar ohne Rückgriff auf irgendwelche erkenntnistheoretische Voraussetzungen eingeführt wird, plausibel machen.

Der vierte und abschließende Teil ist der Frage der Wahrheitssensitivität rationaler Selbstkontrolle bei Descartes und Spinoza gewidmet. Es wird sich zeigen, dass auch im Blick auf dieses Problem erhebliche Unterschiede zwischen den beiden Ansätzen bestehen. Frappant sind insbesondere die Verschiebungen, die sich im Blick auf die Frage ergeben, ob der Verstand ein passives oder ein aktives Vermögen ist. Während er bei Descartes noch als ein passives Vermögen begriffen wird, welches Ideen inklusive ihrer inferentieller Verbindungen gleichsam perzipiert, ${ }^{8}$ distanziert sich Spinoza in seinen späteren Werken von dieser Auffassung. Damit verabschiedet er sich nicht nur vom augustinisch-platonischen Erbe des Kartesianismus, vielmehr macht er als einer der ersten unter den Rationalisten die Sicht frei für eine neue Lösung des Problems, in der nicht nur dem Verstand, sondern auch der Sinnlichkeit eine zentrale Rolle eingeräumt wird.

\section{Descartes über doxastischen Voluntarismus und rationale Enthaltsamkeit}

In der vierten Meditation entwickelt Descartes eine Urteilskonzeption, die bis heute immer wieder für Diskussionen gesorgt hat. Dieser Urteilskonzeption zufolge wirken beim Urteilen intellectus und voluntas so zusammen, dass der Geist kraft seines Willens einer Idee zustimmt, die er zuvor oder gleichzeitig im Intellekt perzipiert hat. Auf dieser Basis erklärt Descartes auch die Möglichkeit von Irrtum. Demnach entstehen Irrtümer

einzig und allein daraus, dass, während der Wille weiter reicht als der Verstand, ich jenen nicht in dessen Grenzen einschließe, sondern ihn auch auf das erstrecke, was ich nicht einsehe. Und da er [der Wille] sich hiergegen unentschieden verhält, so lenkt er leicht vom Wahren und Guten ab, und so irre und sündige ich. ${ }^{9}$

8 Siehe für Descartes v. a. seinen Brief an Regius vom Mai 1641, wo er die intellectio explizit als eine passio mentis definiert und von der aktiven volitio abgrenzt (AT III, 372). Malebranche äussert sich dazu ausführlich im ersten Kapitel von De la Recherche de la Vérité (Euvres I, 23 f.) 9 Die Grundlage der deutschen Zitate wie auch der Referate der verschiedenen Argumente bildet die von Ch. Adam und P. Tannery herausgegebene Werkausgabe (zit. als AT). Unde ergo nascuntur mei errores? Nempe ex hoc uno quod, cum latius pateat voluntas quam intellectus, illam non intra eosdem limites contineo, sed etiam illa quae non intelligo extendo; ad quae cum sit indifferens, facile a vero \& bono deflectit, atque ita \& fallor \& pecco. AT VII, 58. 
Diese Erklärung menschlichen Irrtums hat schon bei Descartes' Zeitgenossen mannigfache Kritik hervorgerufen. ${ }^{10}$ Die Einwände gehen in zwei Richtungen. Auf der einen Seite wurden die konzeptionellen Voraussetzungen Descartes' im Blick auf die Verfasstheit des Willens und dessen Verhältnis zum Verstand in Zweifel gezogen. Kann, so fragt sich, der menschliche Wille tatsächlich so gedacht werden, wie das Descartes' Urteilskonzeption nahelegt, nämlich als ein an sich unbegrenztes Vermögen, das einem an sich begrenzten Verstand gegenüber steht, und das sich je nachdem in der Unentschiedenheit einer Wahlfreiheit oder in der Spontaneität des Zustimmens zu einer als klar und deutlich eingesehenen Idee äußert? ${ }^{11}$

Gegenstand der Kritik bildeten auf der anderen Seite aber auch die erkenntnistheoretischen Voraussetzungen, von denen dieses Modell Gebrauch macht. So hängt Descartes' Strategie der Irrtumsvermeidung u. a. maßgeblich davon ab, dass wir jene wahren Ideen, denen wir Zustimmung leisten dürfen, zweifelsfrei als wahre identifizieren können. Das ist allerdings keineswegs selbstverständlich, sind wir doch, wie Thomas Hobbes in den dritten Einwänden formuliert, sehr oft felsenfest davon überzeugt, dass unsere Auffassungen richtig sind. Zwischen unserer Bereitschaft, einer Idee zuzustimmen, und der Einsicht in ihre Wahrheit besteht daher keineswegs eine so eindeutige Korrelation, wie Descartes meint.

An dieser Stelle könnte man gegen Hobbes einwenden, dass der Zusammenhang, den Descartes' Urteilskonzeption zwischen der Doktrin der eingeborenen Ideen und dem Willen zur Wahrheit voraussetzt, gar kein psychologischer sei. Tatsächlich geht es Descartes nicht darum, eine Relation zu behaupten zwischen dem subjektiven Für-wahr-Halten einer Idee und ihrer Zugehörigkeit zur Klasse der angeborenen Ideen. Was ihn hier interessiert, ist vielmehr, ob sich - immer gesetzt den Fall, es gibt notwendig wahre Ideen, die wir klar und deutlich einsehen können - unser Verhältnis zu unseren Ideen normativen Regeln unterwerfen lässt, die uns in zuverlässiger Weise vor Irrtum schützen. Sein Ziel ist es, mit anderen Worten, den präventiven Regeln seiner Strategie der Irrtumsvermeidung eine kriteriologische Grundlage zu verschaffen.

10 Kritik an Descartes' sogenanntem doxastischem Voluntarismus ist v. a. von Alvin Goldman 1978 geübt worden. Siehe für eine Diskussion v. a. Halbach 2002. In der Descartes-Forschung wird Descartes vor dem Vorwurf eines radikalen doxastischen Voluntarismus meist in Schutz genommen, vgl. Larmore 1984, Rubin 1977, Loeb 1992. Unklar ist jedoch, ob der Wille bei der geforderten Urteilsenthaltung frei ist (siehe dazu Curley 1975, sowie kritisch Cottingham 1988), oder ob umgekehrt lediglich der Zustand der vorangehenden Indifferenz einer der Freiheit ist (vgl. hierzu Halbach 2002, 557).

11 Siehe auch die Einwände von Gassendi zur vierten Meditation in AT VII, 314-318. Wie Haag 2006 gezeigt hat, liegt der kartesischen Urteilskonzeption eine durchaus konsistente Auffassung vom menschlichen Willen zugrunde. 
Diese Stoßrichtung von Descartes’ Überlegungen wird deutlich, wenn man statt seiner Antwort auf Hobbes, die diesbezüglich auch nicht weiterführt ${ }^{12}-$ die Ausführungen ansieht, mit denen er auf einen Einwand von Marin Mersenne reagiert. Wie aus den zweiten Einwänden zu den Meditationen hervorgeht, bereitet Descartes' Urteilskonzeption Mersenne deshalb Unbehagen, weil durch sie indirekt auch „der Türke und jeder beliebige Ungläubige“ von der Sünde, die christliche Religion nicht angenommen zu haben, freigesprochen werden. ${ }^{13}$ Nun kann man das Verhalten eines Ungläubigen in der Tat so beschreiben, dass es den epistemischen Empfehlungen Descartes' entspricht: Der Ungläubige enthält sich der Zustimmung zu einer Idee, von der er keine klare und deutliche Erkenntnis hat.

Wie aus der Antwort von Descartes hervorgeht, teilt er grundsätzlich Mersennes Annahme, dass sich der religiöse Glaube auf an sich dunkle Gegenstände bezieht. Somit fallen die Ideen, die den Gegenstand des christlichen Glaubens bilden, nicht in die Kategorie der klaren und deutlichen Ideen. Er zieht daraus aber differenziertere Schlüsse als Mersenne. So unterscheidet er in einem ersten Schritt zwischen zwei Gesichtspunkten, unter denen unsere Zustimmung zu Ideen betrachtet werden kann: Wir können einerseits nach der Materie oder der Sache fragen, der wir zustimmen sollen, und andererseits nach dem formalen Grund, der uns zur Zustimmung bewegt. ${ }^{14}$ Descartes bezieht sich hier auf eine Unterscheidung, die schon in Thomas von Aquins Erörterung des Glaubens vorkommt. Laut Thomas können wir eine Haltung des Glaubens - oder allgemeiner: kognitive Einstellungen, habitus cognoscitivi - entweder auf ihren Inhalt resp. das, was materialiter erkannt oder angenommen wird, hin betrachten; dann beziehen wir uns auf das materiale Objekt derselben. Oder wir können dieselbe kognitive Einstellung auch daraufhin untersuchen, wodurch sie erkannt bzw. angenommen

12 Descartes weist in seiner Antwort einfach darauf hin, dass es eines sei, etwas klar und deutlich einzusehen, etwas anderes aber, von etwas eine hartnäckige Meinung zu haben, die man ohne einleuchtendes Erfassen in sich aufgenommen habe. Damit unterstreicht er zwar indirekt, dass der Begriff der klaren und deutlichen Idee kein psychologisches Konzept ist, sondern eine erkenntnistheoretische Voraussetzung seiner gesamten Methode ausmacht. Doch wer diese Voraussetzung nicht teilen kann - oder will -, dem hilft Descartes' Antwort auch nicht weiter.

13 Quinto, si nunquam aberrat voluntas aut peccat, cum sequitur claram \& distinctam mentis suae cognitionem, sequ periculo exponat, si conceptum intellectus sectetur minime clare \& distinctum, vide quid inde sequatur: Nempe Turcam, aut alium quemlibet, non solum non peccare quod non amplectatur religionem Christianam, sed etiam peccare si amplectatur, quippe cuius veritatem neque clare neque distincte cognoscat. AT VII, 126.

14 Quippe distinguendum est inter materiam sive rem ipsam cui assentimur, \& rationem formalem quae movet voluntatem ad assentiendum. Nam in hac sola ratione perspicuitatem requirimus. AT VII, 147. 
wird; dann fragen wir nach ihrem formalen Grund. Thomas führt diese Differenzierung im Kontext seiner Auseinandersetzung mit dem genauen Gegenstand des Glaubens an. Konkret geht es um die Frage, ob sich der Glaube ausschließlich auf Gott richtet, oder ob es noch andere Gegenstände des Glaubens gibt wie etwa die Annahme, dass Christus Mensch war. Dank der Unterscheidung von materialem Gegenstand und formalem Grund kann Thomas zeigen, dass man dem Glauben sowohl verschiedene Inhalte zuweisen, als auch Gott als exklusiven Grund für die Annahme derselben auffassen kann. ${ }^{15}$

Das Problem, das Descartes in seiner Antwort auf Mersenne thematisiert, ist ein anderes als jenes von Thomas von Aquin. Zur Diskussion steht nicht mehr der genaue Gegenstand des Glaubens, sondern die Einschätzung der Haltung des Ungläubigen. Gleichwohl ermöglicht die Unterscheidung von Materie und formalem Grund auch Descartes eine differenzierte philosophische Sicht auf Fragen des Glaubens, die ihm ansonsten nicht zur Verfügung stünde. So weist er darauf hin, dass nur im Blick auf den formalen Grund unserer Überzeugungen Klarheit, perspicuitas, gefordert sei. ${ }^{16}$ Was dagegen die Materie selbst betreffe, so führt er fort, bezweifle niemand, dass diese auch dunkel sein könne oder sogar in der Dunkelheit selber bestehen könne, wie etwa wenn wir urteilen, dass alle Dunkelheit aus unseren Begriffen eliminiert werden solle. ${ }^{17}$ Es ist daher nach Descartes durchaus möglich, klare Urteile über dunkle Gegenstände zu bilden.

In einem zweiten, daran anschließenden Schritt führt Descartes eine weitere Unterscheidung ein, nämlich jene zwischen verschiedenen Arten von formalen Gründen. Die Klarheit, die unseren Willen zur Zustimmung bewege, könne von zweifacher Art sein, gehe sie doch einmal vom natürlichen Licht, ein anderes Mal von der göttlichen Gnade aus. Der religiöse Glaube unterscheidet sich somit von den übrigen Urteilen nicht allein aufgrund seiner Materie, sondern v. a. auch durch den besonderen formalen Grund der göttlichen Gnade, die uns zur Zustimmung religiöser Glaubensinhalte bewegt.

Aufgrund dieser Differenzierung kann sich Descartes dann in einem dritten Schritt endlich der Frage annehmen, inwiefern der Ungläubige eine Sünde begehe, wenn er der Idee Gottes nicht zustimme. Descartes' Auffassung, die er überraschend zugleich als kühn und als orthodox charakterisiert, ist dabei folgende: Eine Sünde sei der Unglaube nur deswegen, weil die Ungläubigen sich der göttlichen Gnade widersetzen würden, die auch sie ergriffe, nicht aber des-

15 STh II, 2, q15, art1.

16 Nam in hac sola ratione perspicuitatem requirimus. AT VII, 147. Unklar ist, ob diese Einschränkung für alle oder nur für an sich dunkle Ideen gilt.

17 Et quantum ad materiam, nemo unquam negavit illam esse tollendam, ut absque ullo errandi periculo ipsis assentiri possimus, de hac ipsa obscuritate clarum judicium formo. AT VII, 147. 
wegen, weil sie einer an sich dunklen Idee die rationale Zustimmung verweigern. Im Gegenteil, so fährt er fort,

behaupte [ich] kühn, dass ein Ungläubiger, der von aller übernatürlichen Gnade verlassen und in völliger Unkenntnis darüber wäre, dass unser Christenglauben von Gott geoffenbart ist, und der dennoch dieselben Glaubenssätze, obwohl ihm selbst dunkel, infolge irgendwelcher falschen Gedankengänge annähme, darum nicht gläubig werden würde, sondern vielmehr darin sündigen würde, dass er seinen Verstand nicht richtig gebraucht. Und ich glaube, dass kein rechtgläubiger Theologe jemals hierüber anders gedacht hat. ${ }^{18}$

Ich möchte es hier dahingestellt sein lassen, ob tatsächlich kein rechtgläubiger Theologe jemals anders über die Sünde des Unglaubens gedacht hat. Doch auch unabhängig davon stellt sich die Frage, wie diese Entgegnung Descartes' auf Mersennes Einwand einzuschätzen ist.

Wichtig scheint mir dabei das Folgende zu sein: Descartes geht offenbar davon aus, dass wir auch in Bereichen, in denen wir nicht über klare und deutliche Einsichten verfügen können, eine Verantwortung für unsere Überzeugungen tragen. Ansonsten käme der Metapher des Sündigens jeglicher, auch metaphorische Sinn abhanden. Diese Verantwortung beschränkt sich allerdings nicht darauf, dass wir zu den richtigen Ideen Zustimmung leisten. Entscheidend ist vielmehr, aus welchen Gründen wir das tun. Als gute Gründe können nur die göttliche Gnade oder die Vernunft gelten, nicht aber die Anpassung an eine herrschende Kultur oder der Gehorsam gegenüber der Tradition. Letztere Motive können nach Descartes zwar gute Gründe für bestimmte Handlungen bilden, nicht aber für das Haben von Überzeugungen. Da im Bereich religiöser Ideen die materiale Klarheit wegfällt, und es ferner denkbar ist, dass ein Ungläubiger von jeglicher göttlichen Gnade verlassen ist, kann der Fehler des Ungläubigen auch darin bestehen, dass er wider die Vernunft seine Zustimmung zu religiösen Ideen gibt. ${ }^{19}$

18 Nec sane Turcae aliive infideles ex eo peccant, cum non amplectum religionem Christianam, quod rebus obscuris, ut obscurae sund, nolint assentiri, sed vel ex eo quod divinae gratiae interius illos moventi repugnent, vel quod, in aliis peccando, se gratia reddant indignos. Atque audacter dico infidelem qui, omni gratia supernaturali destitutus, \& plane ignorans ea quae nos Christiani credimus a Deo esse revelata, ipsa tamen, quamvis sibi obscura, falsis aliquibus ratiociniis adductus amplecteretur, non ideo fore fidelem, sed potius in eo peccaturum, quod ratione sua non recte uteretur. Nec puto ullum orthodoxum Theologum aliter unquam de his sensisse. AT VII, 148.

19 Man kann sich hier fragen, ob es sich bei Descartes' Rede von der Sünde wirklich um eine Metapher handelt. Ich meine, dass genau darin eine der subversiven Pointen von Descartes' Antwort liegt. Während nämlich Mersenne sich als Theologe darüber besorgt zeigt, dass wir den Unglauben auf der Basis der Vorgabe von Descartes Erkenntnistheorie nicht mehr als Sünde wider Gott verstehen könnten, taucht der Ausdruck der Sünde in der Antwort von Descartes lediglich als Metapher für jenen rein epistemischen Fehler auf, den jemand begeht, der - im Falle des Ausbleibens jeglicher Gnade - im Widerspruch zum natürlichen Licht handelt. 
Obwohl es also der Wille ist, der das Urteil durch die Zustimmung zu einer bestimmten Idee vollzieht, besteht der Fehler, den jemand begeht, der einer dunklen Idee zustimmt, ohne dass ihn die Gnade dazu bewegt, nicht in einer Kompetenzüberschreitung des Willens, sondern in einem falschen Gebrauch der Vernunft. Demnach trägt für die Irrtümer, die jemand begeht, wenn ihn die göttliche Gnade verlassen hat, auch die Vernunft und nicht etwa der Wille die Verantwortung. Im Endeffekt bedeutet dies, dass der Verstand im Rahmen von Descartes' Ansatz in doppelter Hinsicht gefordert ist: ${ }^{20}$ Erstens muss darüber befunden werden, ob es sich bei bestimmten Ideen um klare und deutliche Ideen handelt oder nicht. Zweitens müssen aus diesem Befund normative Forderungen oder Lizenzen abgeleitet werden. Für beide Aufgaben ist nicht der Wille zuständig, denn er ist dafür gleichsam blind. ${ }^{21}$

Zusammenfassend können wir festhalten, dass Descartes’ voluntaristische Urteilskonzeption nicht nur die Möglichkeit einer Einflussnahme auf die eigenen Überzeugungen einräumt, sondern darüber hinaus auch sicherstellt, dass diese Einflussnahme nach dem Maßstab der Vernunft vonstattengeht. Damit ist sicher die erste Voraussetzung für eine Ethik der Überzeugungen erfüllt. Wie weit damit auch die zweite Voraussetzung der Wahrheitssensitivität rationaler Kontrolle erfüllt ist, wird sich noch erweisen müssen.

\section{Spinoza über doxastischen Determinismus und rationale Selbstreflexion}

Wenn Spinoza sich in der Ethik jeweils mit dem Vokabular anderer Philosophen befasst, dann kommt das oft einem Ockham'schen Reinemachen gleich. Zahlreiche begriffliche Differenzen werden eingeebnet oder gar preisgegeben, wobei weder auf traditionelle Gesichtspunkte Rücksicht genommen wird, noch auf jene phänomenologischen Intuitionen, die diese Unterscheidungen motiviert haben dürften.

20 Man könnte sich hier fragen, in welchem Verhältnis die Begriffe des Verstandes, intellectus, und der Vernunft, ratio, zueinander stehen. Ich denke, dass es sich hier um kontextuell bedingte terminologische Varianten zur Bezeichnung desselben Vermögens handelt. Dass Descartes hier von der ratio spricht, dürfte maßgeblich daran liegen, dass an dieser Stelle nicht der Gegensatz zur voluntas, sondern jener zur göttlichen Gnade das Thema ist.

21 Das unterstreicht v. a. auch Malebranche, dessen Urteilskonzeption weitgehend derjenigen Descartes' entspricht und der den Willen als „puissance aveugle“ charakterisiert. Euvres I, 28. 
Ähnlich radikal geht Spinoza auch mit den begrifflichen Voraussetzungen um, die Descartes' Urteilskonzeption zugrunde liegen.22 Mindestens in fünf Punkten sind deutliche Differenzen festzustellen: Erstens hat nach Spinoza der menschliche Geist keinen absoluten und freien Willen, sondern der Mensch ist in seinem Wollen immer schon determiniert. Überhaupt ist nach Spinoza die Annahme eines Vermögens des Willens hinter den einzelnen voluntativen Akten nicht haltbar (2p48). ${ }^{23}$ Zweitens lehnt Spinoza die Annahme eines vom Haben von Ideen verschiedenen voluntativen Akts ab. Dass wir zu einer Idee, die wir perzipieren, erst noch zustimmen müssten oder könnten, ist für Spinoza blanker Unsinn (2p49). Davon ausgehend weist er drittens jegliche Differenzierung zwischen intellektuellen und voluntativen Akten zurück (2p49dem). Wenn wir eine Idee haben, dann ist mit der Einsicht in den Gehalt dieser Idee schon gegeben, wie wir uns zu ihr verhalten. Viertens ebnet Spinoza die Unterscheidung zwischen angeborenen, erworbenen und fiktiven Ideen ein, was im Endeffekt auf eine radikale Kritik am kartesischen Innativismus hinausläuft. ${ }^{24}$ Fünftes ist schließlich darauf hinzuweisen, dass es vor dem Hintergrund des Ideenholismus, den Spinoza gemäß zahlreichen Interpretationen vertreten hat, ${ }^{25}$ ziemlich

22 Siehe dazu auch Della Rocca 2003.

23 Auf Textstellen aus der Ethik wird gemäss der in der angelsächsischen Literatur eingebürgerten Zitierweise verwiesen. „2p48s“ rekurriert demnach auf das Scholium von Proposition 48 des zweiten Teils der Ethik.

24 Diese Einschätzung scheint kontroverser als sie ist. Dass Spinoza sich von Descartes' Klassifikation von Ideen entfernt, zeigen auf der einen Seite Delahunty 1985, 24, und Allison 1987, 114; Deleuze 1968, 134, behauptet, dass Spinozas grundlegende Intuitionen empiristischer Natur seien. Dem stehen scheinbar Wilson 1996, 137, n. 36, Garrett 2003, 82, sowie Marhall 2008, 82-85, gegenüber, denen zufolge es in Spinozas Ansatz durchaus Raum für die Annahme angeborener Ideen gibt. Festzuhalten ist, dass die Ethik nicht von ideae innatae spricht, und im Tractatus de intellectus emendatione ist nur von der vis nativa und den instrumenta innata des Verstandes die Rede (vgl. TIE §31 f.). Ferner ist zu beachten, wie Spinoza in 2p49d und 2p49s von den Beispielen der Idee des Dreiecks und der Idee des Pegasus Gebrauch macht, die Descartes in seiner Antwort auf Caterus zur genaueren Illustration der Differenz zwischen angeborenen und fiktiven Ideen heranzieht. Spinozas erklärt das Haben dieser Ideen auf eine Weise, welche die kartesische Klassifikation unterminiert. Das schließt nicht aus, dass sich Spinoza von Descartes' Konzeption der angeborenen Idee inspirieren ließ, vgl. dazu meine Ausführungen in Renz 2010, 105-109, doch werden dadurch all jene Interpretationen in Frage gestellt, die Spinozas Ansatz undifferenziert in die Nähe eines kartesischen Innativismus rücken. Das tun allerdings weder Wilson, Garrett noch Marshall. Somit verlagert sich die Kontroverse bezüglich Spinozas Innativismus auf andere Fragen, nämlich erstens, was nach Spinoza heißen kann, eine angeborene Idee zu haben, und zweitens, wovon menschliche Subjekte angeborenes Wissen haben können. Von diesen Debatten ist die obige Einschätzung, die einzig das Verhältnis von Spinoza zu Descartes' Klassifikation zum Gegenstand hat, nicht betroffen. Vgl. dazu aber Anm. 43.

25 Vgl. für den Holismus Spinozas v. a. Della Rocca 1996, 44-83, sowie Perler 2008. 
sinnlos ist, anzunehmen, wir hätten es je mit Ideen zu tun, die so einfach wären, dass wir von ihnen eine klare und deutliche Erkenntnis hätten. ${ }^{26}$

Angesichts der Ausmaße dieses Kahlschlags stellt sich die Frage, was in Spinozas Ansatz von der kartesischen Ethik der Überzeugungen noch übrig bleibt. Tatsächlich wird man zunächst einmal die Unterschiede betonen müssen. Anders als Descartes vertritt Spinoza einen doxastischen Determinismus, demzufolge unsere Überzeugungen weitgehend von Ursachen bestimmt sind, auf die wir keinen unmittelbaren Einfluss haben. ${ }^{27}$ Davon betroffen sind nicht nur die Inhalte der Ideen, die wir perzipieren, sondern auch unsere affirmativen oder negativen Einstellungen dazu. Spinoza zufolge können wir uns der Zustimmung zu unseren Ideen nicht einfach enthalten, wie das die kartesische Urteilskonzeption nahelegt. Denn seiner Auffassung nach sind selbst unsere Fiktionen, in denen wir dem phänomenologischen Anschein nach genau das tun, durch psychologische Mechanismen bestimmt, auf die wir nur bedingt Einfluss haben. ${ }^{28}$ Da wir das oft nicht durchschauen, können wir zudem meist gar nicht mit Sicherheit sagen, woher unsere Neigung, einer Idee zuzustimmen, rührt. Das zeigt, dass Spinoza in dem Moment, wo er von Descartes' Unterscheidung zwischen angeborenen, erworbenen und fiktiven Ideen Abstand nimmt, auch jene heuristischen Kriterien preisgibt, die es Descartes erlaubt haben zu entscheiden, ob wir einer Idee zustimmen dürfen. Wir müssen daher nach Spinoza darauf gefasst sein, dass im Akt des Urteilens - oder genauer: des Habens von Ideen - psychologische Gesetzmäßigkeiten ins Spiel kommen, die das Subjekt in einer für es selber undurchschaubaren Art und Weise steuern.

Dennoch gibt es einen Punkt, in dem Spinoza und Descartes sich einig sind, und dieser ist für die rationalistische Ausrichtung der Ethik entscheidend. Trotz seiner Ablehnung der kartesischen Klassifikation von Ideen hält Spinoza im Prinzip an der Unterscheidung zwischen guten und schlechten Gründen fest oder anders gesagt: zwischen Ideen, die auf logischen Einsichten beruhen, und

26 Siehe auch Renz 2010, 101. Spinozas Holismus schließt nicht aus, dass wir adäquate Ideen haben können, die genauso notwendig wahr sind wie Descartes' klare und deutliche Ideen. Doch in der Erklärung dieser Möglichkeit kann man sich nicht an Descartes’ Konzeption der klaren und deutlichen Ideen orientieren. Vgl. dazu auch meine Ausführungen zur intuitiven Erkenntnis in Renz 2010, 290-297.

27 Wie in Anmerkung 32 noch deutlich wird, nehme ich nicht an, dass Spinoza von einem strikten Nezessitarismus ausgeht, wonach es für unsere Überzeugungen nur eine mögliche Kausalgeschichte gibt. Dies ist nicht zuletzt für die Unterscheidung zwischen guten und weniger guten Gründen der Zustimmung zu einer Idee von Belang, kann man doch fragen, ob diese Unterscheidung vor dem Hintergrund eines strikten Nezessitarismus wirklich sinnvoll ist.

28 Das illustriert u. a. das Beispiel des sich ein geflügeltes Pferd vorstellenden Knaben in 2p48s. Siehe dazu auch Anmerkung 24 sowie Renz 2010, $107 \mathrm{f}$. 
solchen, die sich dem Einfluss psychologischer Gesetzmäßigkeiten verdanken. So macht es für ihn bekanntlich einen wesentlichen Unterschied, ob jemand der Proposition , $2+2=4$ “ deshalb zustimmt, weil es ihm ein anderer gesagt, oder aber deshalb, weil er die Regeln kennt und richtig gerechnet hat. ${ }^{29}$ Dass Spinoza diesen Unterschied zwischen logischen Gründen und psychologischen Ursachen anerkennt und ihm einen epistemischen Wert zuspricht, trennt ihn nicht zuletzt von Hobbes, der in seinen Einwänden gegen die Meditationen zwar Spinozas Vorbehalte gegen die kartesische Einteilung sämtlicher Ideen in angeborene, erworbene und fiktive Ideen vorweggenommen hat, aber in seiner eigenen mechanistischen Erklärung des Denkens die Unterscheidung von Logik und Psychologie preisgibt.

Doch wie, so fragt sich, kann man diesen Unterschied zwischen epistemisch höherwertigen, logisch hergeleiteten Überzeugungen und solchen, deren Entstehung auf psychologische Ursachen zurückzuführen ist, auf der Basis von Spinozas Ansatz überhaupt plausibel machen? Worin ist er begründet, und woran kann man ihn festmachen?

An dieser Stelle ist ein Blick auf die Unterscheidung von adäquaten und inadäquaten Ideen zu werfen. Spinoza verwendet die Adjektive „,adäquat“ bzw. „,inadäquat“ in der Ethik in zwei Kontexten. Im zweiten Teil der Ethik werden damit Wahrnehmungen, Erkenntnisse oder allgemeiner: epistemische Prozessen oder Zuständen auf ihren epistemischen Wert hin qualifiziert. Die Grundlage dazu wird mit 2def4 explizit, wo Spinoza klar macht, dass er von einer Korrelation zwischen der Adäquatheit und der Wahrheit von Ideen ausgeht. Gleichzeitig hält Spinoza explizit fest, dass es sich dabei um zwei verschiedene Eigenschaften von Ideen handelt: Während die Wahrheit einer Idee in deren Übereinstimmung mit ihren (extramentalen) Gegenständen besteht, ist ihre Adäquatheit von „inneren Merkmalen oder Eigenschaften“ abhängig. ${ }^{30}$ Das darf man nun allerdings nicht so verstehen, dass Adäquatheit eine Eigenschaft von einzelnen isolierten Ideen ist. Wäre dies der Fall, so könnte man nicht erklären, wie es für Spinoza einen Unterschied machen kann, ob ein Kleinkind oder aber der mit natürlichen Zahlen und den basalen Rechenregeln vertraute Vater den Gedanken „2 $+2=4$ “ hat. Offensichtlich macht es für den Grad der Adäquatheit einer Idee einen Unterschied, wer diese Idee hat. Wie ist das nun mit der Bestimmung der Adäquatheit

29 Vgl. das Beispiel der Zahlenreihe mithilfe dessen in 2p40s2 die Unterscheidung der drei Erkenntnisgattungen illustriert wird. Das obige Rechenbeispiel ist etwas einfacher, doch die Pointe bleibt dieselbe.

30 Per ideam adaequatam intelligo ideam, quae, quatenus in se sine relatione ad objectum consideratur, omnes verae ideae proprietates sive denominationes intrinsecas habet. Explicatio. Dico intrinsecas, ut illam secludam, quae extrinseca est, nempe convenientiam ideae cum suo ideato. 2def4. Ethik, 100. 
als einer intrinsischen Eigenschaft von Ideen zu verbinden? An dieser Stelle ist erstens daran zu erinnern, dass Spinoza in 2p11 den Geist einzelner Menschen mit Ideen gleichsetzt. Zweitens ist darauf hinzuweisen, dass sich nach Spinoza der Geist eines Menschen qualitativ vom Geist eines andern dadurch unterscheidet, dass er andere Ideen enthält. Das legt nahe, dass es bei der Unterscheidung von adäquaten vs. inadäquaten Ideen maßgeblich darauf ankommt, in was für Relationen eine Idee zu andern Ideen steht, die dasselbe Subjekt hat.

Es bleibt die Frage, wie genau die Relationen beschaffen sein müssen, welche die Adäquatheit einer Idee ausmachen. Dazu ist ein Blick auf die zweite Verwendungsweise des Vokabulars der Adäquatheit hilfreich. Die Adjektive „adäquat“ und ,inadäquat“ werden nämlich in der Ethik auch dazu gebraucht, um die vollständige Ursache einer bestimmten Wirkung von bloß partiellen Ursachen zu unterscheiden. Blickt man nun auf den genauen Wortlaut von 3def1, wo diese Verwendungsweise eingeführt wird, so ist frappant, dass die Unterscheidung von adäquaten vs. inadäquaten Ursachen ebenfalls in epistemischen Zusammenhängen zum Tragen kommt, und das, obwohl es sich beim Begriff der Kausalität selber um ein metaphysisches Problem handelt, das bereits im ersten Teil der Ethik abgehandelt wird. Wie hier Spinoza definitorisch festhält, ist ein Ding $\mathrm{x}$ genau dann eine adäquate Ursache von einem Ding $y$, wenn man allein aufgrund der Idee von $\mathrm{x}$ eine klare und deutliche Idee von y haben oder bilden kann. ${ }^{31}$ Es geht also bei der Frage nach der Adäquatheit oder Inadäquatheit einer Ursache wesentlich darum, ob die inferentiellen Beziehungen zwischen zwei Ideen derart sind, dass die Kenntnis einer Ursache eine vollständige Erkenntnis ihrer Wirkung erlaubt oder nicht. Letzteres ist der Fall, wenn jemand, der über eine Kenntnis der basalen Rechenregeln verfügt, aus zwei gegebenen Zahlen eine dritte berechnet. Anders verhält es sich, wenn ein Kind darauf konditioniert ist, die Frage seines Vaters nach dem Resultat einer bestimmten Rechenaufgabe in einer Weise zu beantworten, wie er es erwartet. Dieses Kind kann zwar die Frage „Was gibt $2+2$ ?" wahrheitsgemäß beantworten. Insofern ist auch seine Antwort teilweise durch die Frage verursacht, doch es ist dem Kind nicht möglich, seine Antwort als vollständig durch die Frage verursacht zu begreifen. Es wird sie womöglich geben in der Meinung, bloß geraten zu haben.

Wir können somit festhalten, dass es für Spinoza ähnlich wie für Descartes einen Unterschied ausmacht, ob eine Überzeugung auf guten Gründen beruht oder aber das relativ beliebige Produkt eines psychologischen Prozesses ist. Wie

31 Causam adaequatam appello eam, cujus effectus potest clare et distincte per eandem percipi. Inadaequatam autem seu partialem illam voco, cujus effectus per ipsam solam intelligi nequit. 3def1, Ethik 222. 
Descartes geht Spinoza ferner davon aus, dass diese Unterscheidung für den epistemischen Wert einer Überzeugung wesentlich ist. Genau an dieser Unterscheidung, so meine These, macht sich daher auch Spinozas Variante einer Ethik der Überzeugungen fest. Sollte es uns nämlich möglich sein, zu bewirken, dass wir vermehrt adäquate Ideen haben - was gleichbedeutend damit ist, dass wir gut begründete und epistemisch wertvollere Überzeugungen bilden -, so wird dies aufgrund der Korrelation der Adäquatheit von Ideen mit ihrer Wahrheit auch die veridische Bilanz unserer doxastischen Tätigkeiten verbessern.

Dass diese Möglichkeit überhaupt besteht, ist nun vor dem Hintergrund der oben erörterten Kritik an Descartes alles andere als selbstverständlich. So dürfte es Spinoza für zwecklos halten, regulative Prinzipien aufzustellen, deren Ziel es wäre, Irrtümer präventiv auszuschließen. Das würde nicht nur voraussetzen, dass wir - ähnlich wie Descartes mit seiner Klassifikation von angeborenen, erworbenen und fiktiven Ideen - über allgemeine heuristische Kriterien verfügen, die uns gleichsam vorweg anzeigen, ob wir eine Idee akzeptieren sollen oder nicht. Ferner müssten wir, um von solchen Kriterien Gebrauch machen zu können, im Stande sein, uns der Zustimmung zu einer Idee zu enthalten. Doch das ist im Rahmen von Spinozas Ansatz keine Option. Seinem doxastischen Determinismus zufolge können wir uns, wenn uns eine Idee einmal vor Augen steht, gar nicht mehr willentlich zu ihr verhalten. Zudem setzt die Anwendung seiner Unterscheidung von adäquaten und inadäquaten Ideen voraus, dass wir eine Idee genau daraufhin untersucht haben, wie sie zustande gekommen ist. Es wäre daher seiner Auffassung nach illusorisch zu meinen, wir könnten Irrtümer mit so einfachen methodischen Regeln vermeiden, wie das Descartes vorgeschlagen hat (und wie das nach ihm etwa auch Antoine Arnault und Pierre Nicole in ihrer Logique ou l'art de penser weitergeführt haben).

Gleichwohl schließt Spinoza m. E. nicht völlig aus, dass wir einen gewissen Einfluss darauf haben, welche Überzeugungen wir haben. Oder genauer: Wir können heute einen Einfluss darauf nehmen, welche Überzeugungen wir morgen haben werden. ${ }^{32}$ Dazu müssen wir aus der Reflexion auf die Genese unserer heu-

32 Ein anonymer Gutachter hat an dieser Stelle moniert, dass ich Spinozas metaphysischen Nezessitarismus vernachlässige. Tatsächlich stellt die Annahme eines Nezessitarismus für die Behauptung eines - nota bene: indirekten - Einflußes auf unsere zukünftigen Überzeugungen ein Problem dar. Für den strikten Nezessitaristen gibt es grundsätzlich nur eine reale Möglichkeit, wie morgen die Welt aussehen kann. Daraus folgt zwar nicht zwingend, dass die Rede von einem Einfluss auf unsere zukünftigen Überzeugungen gegenstandslos wird, denn wir können mindestens aus subjektiver Perspektive den Eindruck haben, dass wir uns im einen Fall anstrengen, im andern Fall nicht. Doch das Resultat ist in beiden Fällen dasselbe, und es stellt sich daher die Frage, ob vor dem Hintergrund einer nezessitaristischen Spinoza-Interpretation nicht auch die Unterscheidung zwischen adäquaten und inadäquaten Ideen relativiert wird. Das wäre 
tigen Überzeugungen epistemisches Kapital schlagen. Genau an dieser Stelle wird die Unterscheidung von logischen Gründen und psychologischen Gesetzen wichtig. Denn wenn wir uns auch nicht zu bestimmten Überzeugungen entschließen können, so können wir doch die Überzeugungen, die wir haben, daraufhin untersuchen, woher sie ihre Überzeugungskraft haben. Im Zuge einer solchen Untersuchung kann es durchaus geschehen, dass wir zu dem Schluss kommen, dass sich die vermeintlich innere Überzeugungskraft einer Idee lediglich externen Ursachen verdankt, was zur Folge hat, dass von ihrer ehemaligen Plausibilität nicht mehr viel übrigbleibt. ${ }^{33}$

Eine solche Reflexion ist natürlich kein Allheilmittel. Erstens ist sie nicht in allen Fällen sinnvoll. Wie Descartes kennt auch Spinoza den pragmatischen Nutzen von Wissen an, das wir rein aus Überlieferung haben. Zweitens wird durch eine solche Reflexion die Macht von psychologischen Mechanismen nicht ein für alle Mal ausgeräumt. Doch wir können uns mindestens in der Reflexion zu ihnen verhalten. Wir können etwa jene unserer Ideen, die unser Denken nachhaltig prägen und an denen wir möglichweise auch leiden, auf ihre Konsistenz und Herkunft befragen. Und es ist klar: Wenn wir dies tun, dann werden diejenigen Ideen, die wir als Effekt psychologischer Mechanismen durchschauen, in ihrer Überzeugungskraft geschwächt, während umgekehrt jene, für die wir gute Gründe entdecken, an Plausibilität gewinnen.

Wir können somit festhalten, dass auch im Rahmen von Spinozas doxastischem Determinismus eine gewisse Einflussnahme des Subjekts auf seine eigenen Überzeugungen denkbar ist. Die Frage stellt sich allerdings, ob es dazu nicht noch weiterer, bislang ungenannter Voraussetzungen bedarf. Tatsächlich scheint mir, dass Spinoza mindestens Folgendes annehmen muss: Er muss dem menschlichen Geist zugestehen, dass in der Reflexion auf die eigenen Überzeugungen das rationale Verstehen die Führung übernehmen kann. Denn wenn das epistemische Ziel tatsächlich darin besteht, dass wir deutlicher zwischen unseren inhaltlich begründeten Auffassungen und den aufgrund von psychologischen Mechanismen zustande gekommenen Überzeugungen unterscheiden, dann kann

für die hier vorgeschlagene Lesart natürlich fatal - aber nicht nur für sie. Auch nezessitaristische Lesarten machen von dieser Unterscheidung Gebrauch, etwa wenn es darum geht, den Vorwurf des Fatalismus abzuwenden, vgl. z. B. Perler 2011, 379. Mir scheint es daher wichtig festzuhalten, dass in der Forschung nach wie vor umstritten ist, ob Spinoza wirklich einen strikten Nezessitarismus vertreten wollte. Dafür argumentieren u. a. Garrett 1991, Nadler 2006, 106-8, Perler 2006, 59-61 und 2011, 376; dagegen argumentieren Schütt 1985, Curley/Walsky 1999, sowie kürzlich Martin 2010 und ich selber in Renz 2009a, 81-85, sowie Renz 2010, 60 f.

33 Hampe $(2004,239)$ hat diesen Vorgang als eine „Dekonditionierung des menschlichen Geistes" beschrieben, im Zuge derer unsere kontingenten Bewertungen und Musterbilder aufgelöst werden. 
die Reflexion nicht einfach so ablaufen wie das psychoanalytische freie Assoziieren auf der Couch. Solches Assoziieren mag eine heuristische Funktion haben: Wir können dadurch entdecken, welche assoziativen Verknüpfungen zwischen Inhalten unser Denken de facto leiten. Doch wenn die dabei erinnerten Assoziationsketten ihre determinierende Kraft verlieren sollen, dann darf es nicht bei der Evokation von habitualisierten assoziativen Verbindungen bleiben, sondern es bedarf auch einer kritischen Evaluation im Lichte der Unterscheidung von logischen Gründen und psychologischen Mechanismen. Erst die erkenntnistheoretische Analyse unserer Ideen und ihrer Verbindungen kann uns eine gewisse Distanz gegenüber unseren unwillkürlichen Impulsen zur Bejahung bestimmter assoziationsgeleiteter Schlüsse verschaffen. Spinozas Erkenntnistheorie kann, mit anderen Worten, nur dann in eine Ethik der Überzeugungen münden, wenn sie Raum für eine Form der Reflexion lässt, die selber kein passives Erleiden ist; ansonsten wäre nicht einzusehen, wie der natürliche Hang, sich dem Assoziieren zu überlassen, überwunden werden kann.

Es ist unter anderem vor diesem Hintergrund $\mathrm{zu}$ verstehen, dass im dritten Teil der Ethik zwischen zwei Arten der Affekte unterschieden wird, nämlich einerseits den actiones, denen vollständig begründete Einsichten zugrunde liegen, und andererseits den passiones, die durch externe Umstände mit verursacht sind (3def1-3). ${ }^{34}$ Ich denke, dass diese Unterscheidung verallgemeinert und auf alle mentalen Zustände angewendet werden kann, also auch auf solche, die nicht emotionaler Art sind und nicht unter die Kategorie der Affekte fallen. Damit beruht, was bisweilen als Grundlage von Spinozas Machttheorie aufgefasst wird, schlicht und einfach auf der Unterscheidung zwischen rationalen und mit den Inhalten unseres Denkens verbundenen Gründen einerseits und inhaltsindifferenten und auf rein psychologischen Mechanismen basierenden Ursachen andererseits. Der Grundgedanke ist denkbar einfach: Wer eine bestimmte Überzeugung als bloßes Produkt von psychologischen Mechanismen entlarvt, der wird Mühe haben, sich als ihr Urheber zu begreifen. Wer hingegen die rationalen Gründe einer Überzeugung kennt und einsieht, der ist in gewisser Weise im Besitz der Ursache dieser Überzeugung, und das, obwohl er nicht anders kann, als diese Überzeugung zu haben..$^{35}$

34 Der genaue Gedankengang an dieser Stelle ist ziemlich kompliziert, und zwar zum einen deshalb, weil er in den drei Definitionen, die er seiner Affektenlehre voranstellt, nicht einfach neue Begriffe einführt, sondern sich implizit auf frühere Ausführungen stützt. Zum anderen bauen auch die drei Definitionen aufeinander auf.

35 Damit verbunden ist eine weitere Annahme, auf die ich hier nur am Rande hinweisen kann: Spinoza ist offensichtlich der Auffassung, dass rationale Gründe auch Ursachen sind. Das ist entscheidend für die machttheoretischen Implikationen seiner Ethik der Überzeugungen: Wer 
Insgesamt können wir somit festhalten, dass Spinoza zwar etliche der kartesischen Vorgaben über Bord wirft, aber mit der Unterscheidung zwischen den rationalen Gründen und psychologischen Ursachen von Ideen dennoch auf eine Ethik der Überzeugungen aus ist. Man könnte auch sagen: Er distanziert sich von Descartes' psychologischem Apparat, bleibt aber dessen rationalistischen Intentionen treu.

\section{Descartes' und Spinozas Antworten auf das Problem der Wahrheitssensitivität von Überzeugungen}

Die vorangegangenen Ausführungen haben gezeigt, dass nicht nur in Descartes' voluntaristischem, sondern auch in Spinozas deterministischem Ansatz die erste Voraussetzung für eine Ethik der Überzeugungen erfüllt ist. Beide räumen die Möglichkeit einer rationalen Einflussnahme auf die eigenen Überzeugungen ein, allerdings funktioniert diese unterschiedlich. Während sie bei Descartes dadurch erfolgt, dass die Vernunft es dem Willen entweder gestattet oder verbietet, bestimmte Ideen zu bejahen, geht es bei Spinoza darum, die Ideen, die man hat, einer kausalen Analyse zu unterziehen, mit dem Resultat, dass diejenigen Auffassungen, die sich lediglich irgendwelchen inhaltsindifferenten psychologischen Umständen verdanken, ihre Überzeugungskraft verlieren.

Wie steht es demgegenüber mit der zweiten Voraussetzung, die oben unter das Stichwort der Aufrechterhaltung der Wahrheitssensitivität unserer Überzeugungen subsumiert wurde? Wie wird im Rahmen von Descartes' und Spinozas Ansätzen sichergestellt, dass die Geist-auf-Welt-Ausrichtung unserer Überzeugungen auch dann aufrechterhalten bleibt, wenn wir auf sie Einfluss nehmen?

Descartes' Antwort mutet zunächst einfach an. Wie bereits angedeutet, geht er davon aus, dass der Verstand ein passives Vermögen ist. Mittels des Verstandes perzipieren wir Ideen, und wer nur richtig „hinschaut“, der weiß sogleich, ob es sich bei einer gegebenen Idee um eine klare und deutliche oder um eine dunkle und verworrene Idee handelt. ${ }^{36}$ Da diese Unterscheidung auch das Krite-

Gründe hat, verfügt über Ursachen, und wer über Ursachen verfügt, der hat die Dinge in seiner Macht. Dafür, Gründe als Ursachen aufzufassen, hat sich in der jüngeren Zeit v. a. Davidson 2001 (zuerst 1963) ausgesprochen, dagegen v. a. Harman 1970.

36 Ich sehe hier der Einfachheit halber davon ab, dass es für Descartes auch klare, aber undeutliche Ideen gibt. Vgl. aber auch Renz 2003. 
rium dafür darstellt, ob einer Idee zugestimmt werden darf oder nicht, ist letztlich auch die gebietende Rolle, welche die Vernunft dem Willen gegenüber übernimmt, in der Passivität des Verstandes begründet.

Diese Antwort steht in klarem Einklang mit zahlreichen Grundannahmen jenes Programms, das Descartes in seinen Meditationen verfolgt. Doch wie befriedigend man sie in der Sache einschätzt, hängt auch davon ab, für wie plausibel man den grundsätzlichen Anspruch der Meditationen hält, ein absolutes Fundament für Wissen zu schaffen. ${ }^{37}$ Wer Bedenken gegenüber dem epistemischen Fundamentalismus Descartes' hat, der wird auch eine reservierte Haltung gegenüber der kartesischen Variante einer Ethik der Überzeugungen einnehmen wollen. So stellt sich insbesondere die Frage, wie wir sicher sein können, dass wir im Wiederaufbau unseres Überzeugungssystems, der auf den radikalen Umsturz der bisherigen Auffassungen folgen muss, die angeborenen Ideen richtig identifizieren. Wie wissen wir, welche unserer Ideen angeborene sind? Oder anders gefragt: Wie wissen wir, ob eine Idee klar und deutlich ist?

Descartes würde an dieser Stelle wohl ähnlich antworten, wie er das in seinen Antworten auf Hobbes getan hat. Wie bereits erwähnt, zieht Hobbes in seinen Einwänden in Zweifel, dass ein Zusammenhang zwischen der Klarheit von Ideen und ihrer Wahrheit besteht. Jeder, der über eine Sache keine Zweifel hege, glaube im Besitz jenes Lichts zu sein, wie es Descartes zufolge jemand habe, der etwas klar einsehe. Dieses Licht könne daher nur die Ursache dafür sein, dass jemand seine Meinung hartnäckig verteidige, nicht aber dafür, dass er wisse, dass sie wahr sei. ${ }^{38}$ Die Eigenschaft der Klarheit und Deutlichkeit von Ideen kann, so die Pointe dieses Einwands, nur die subjektive Gewissheit von Überzeugungen, nicht aber deren objektive Wahrheit selbst verbürgen.

Descartes reagiert auf diesen Einwand, indem er darauf hinweist, dass er mit der Metapher des Lichts die Durchsichtigkeit, perspicuitas, einer Erkenntnis meine. Über dieses Licht würden vielleicht nicht alle verfügen, die es zu haben meinen. Das schließe aber nicht aus, dass durchsichtige Erkenntnisse von einer sturen Meinung, die man ohne evidente Wahrnehmung gefasst habe, grund-

37 Wie Perler 1998, 84 f., zu Recht geltend macht, muss man sich davor hüten, aus den rationalistischen Grundlegungsbemühungen der Meditationen einen Wissensbegriff abzuleiten, der kein empirisches Wissen zuließe. Das schließt indes nicht aus, dass auch die Möglichkeit empirischen Wissens vom Erfolg der rationalistischen Grundlegung abhängig ist.

38 AT VII, $191 \mathrm{f}$. Unusquisque autem qui dubitatione caret, talem lucem praetendit, \& habet propensionem voluntatis id de quo non dubitat, non minorem quam qui revera scit. Potest ergo lux haec esse causa quare quis obstinate opinionem aliquam defendat vel teneat, et non quod sciat veram eam esse. 
sätzlich verschieden seien. ${ }^{39}$ Descartes hält also auch angesichts von Hobbes' Einwand an der Unterscheidung zwischen logischen Gründen und psychologischen Ursachen fest. Anders als Hobbes' Einwand unterstellt, hat die Gewissheit, welche klare und deutliche Ideen auszeichnet, einen epistemischen und nicht bloß psychologischen Ursprung.

Wie befriedigend ist diese Antwort? An dieser Stelle ist zu berücksichtigen, dass Hobbes nicht danach fragte, was klare und deutliche Ideen sind, sondern wie wir sie erkennen und von bloß subjektiv gewissen Ideen unterscheiden können. Wie können wir insbesondere ausschließen, dass wir in diesem Punkt einer Selbsttäuschung unterliegen? Für Descartes ist klar, dass wir, wenn wir eine klare und deutliche Idee haben, diese auch als eine solche identifizieren können. Doch lässt sich damit Hobbes' Sorge wirklich zerstreuen? Tatsächlich könnte man sagen, dass es dazu nicht ausreicht, klare und deutliche Ideen als solche $\mathrm{zu}$ identifizieren, sondern dass wir überdies ein Mittel benötigen, um jene Ideen auszusondern, die nicht klar und deutlich sind, von deren Richtigkeit wir aber gleichwohl subjektiv felsenfest überzeugt sind. Nun würde allerdings Descartes an dieser Stelle wohl antworten, dass das im Rahmen seines Ansatzes gar kein echtes Problem mehr ist. Denn hat man sich - wie er es in der Zweifelsmeditation vorexerziert hat - erst einmal aller bezweifelbaren Meinungen entledigt, so muss man keine fälschlicherweise für wahr gehaltenen Ideen mehr entlarven. Stattdessen gehe es nur mehr darum, als fraglich anerkannte Ideen daraufhin zu überprüfen, ob sie jene perspicuitas aufweisen, die klare und deutliche Ideen epistemisch auszeichnen.

Wir können somit festhalten, dass Descartes' Konzeption der wahren und deutlichen Ideen mindestens für jene Leser seiner Meditationen überzeugend sein müsste, die ihm durch die erste und zweite Meditation hindurch gefolgt sind. Allerdings ist damit sein epistemischer Fundamentalismus noch nicht vor sämtlichen Einwänden sicher. Denn wenn wir auch dem Begriff der klaren und deutlichen Ideen jene Funktion zugestehen, die Descartes ihm zuweist, so kann man immer noch Zweifel an der Art und Weise hegen, wie er diesen Begriff im Zusammenhang mit der Idee Gottes zur Anwendung bringt. Dass diese Idee unter die klaren und deutlichen Ideen fällt, ist für Descartes von entscheidender Bedeutung, denn von ihr hängt auch die Klarheit und Deutlichkeit sehr vieler anderer Ideen ab. Nun dürfte diese Einschätzung nicht ohne Widerspruch zur Kenntnis genommen worden sein, und wie wir oben gesehen haben, gibt es auch Descar-

39 AT VII, 192. Nemo enim nescit per lucem in intellectu intelligi perspictuitatem cognitionis, quam fortenon habent omnes qui putant se habere; sed hoc non impedit quominus valde diversa sit ab obstinate opinione absque evidenti perception concepta. 
tes zufolge mindestens gewisse Aspekte dieser Idee, die Sache des Glaubens und mithin dunkel sind. Wir können daher festhalten, dass Descartes' Ethik der Überzeugungen trotz der liberalen Antwort auf Mersennes Einwände gegen die vierte Meditation nur funktioniert, wenn man der Idee Gottes jenen Status zugesteht, die ihr in der dritten Meditation zugesprochen wird.

Im Unterschied zu Descartes, setzt sich Spinoza in seiner Ethik von jeglichem augustinisch-platonischen Einfluss radikal ab. Das geht sehr schön aus der Definition von idea im zweiten Buch der Ethik hervor (2def3). Spinoza bestimmt hier das Haben von Ideen explizit als ein Formen von Begriffen und nicht als ein Wahrnehmen. In der anschließenden Erklärung erläutert er diese Unterscheidung ferner dahingehend, dass der Ausdruck perceptio andeute, dass der Geist etwas erleide, während der Begriff des conceptus eine Handlung des Geistes ausdrücke (2def2expl).

Inwiefern tangiert dies das Problem der Aufrechterhaltung der Wahrheitssensitivität von Überzeugungen?

Prima facie scheint es, als sei auf der Basis dieser Definition die Forderung nach der Erhaltung der für das Erkennen konstitutiven Geist-auf-Welt-Ausrichtung schwieriger zu erfüllen. Folgt man beispielsweise John Searles Überlegungen zur Intentionalität sinnlicher Erfahrungen, so scheint es für die Geist-aufWelt-Ausrichtung unserer Wahrnehmung wesentlich $\mathrm{zu}$ sein, dass uns seitens des intentionalen Gegenstandes etwas widerfährt. ${ }^{40}$ Wenn das stimmt - und es spricht einiges dafür, dass an Searles Argumentation etwas dran ist,$-{ }^{41}$ dann liegt der Schluss nahe, dass die Gefahr, Opfer des eigenen Wunschdenkens zu werden, umso grösser wird, je mehr unsere Ideen als Ausdruck unserer eigenen Aktivität begriffen werden. Erst recht virulent erscheint diese Gefahr, wenn man sich vergegenwärtigt, welche unbewussten Prozesse Spinozas Theorie der imaginatio ${ }^{42}$ zufolge an der Bildung unserer Überzeugungen beteiligt sind. Da sind nicht nur kontingente Assoziationen, sondern auch gänzlich zufällige Kausalattribuierungen, Projektionen oder Imitationen von Wünschen und Affekten am Werk. Kann, so fragt sich, angesichts eines solchen Wildwuchses von Ideen überhaupt noch von der Wahrheitssensitivität von Überzeugungen die Rede sein?

40 Siehe etwa Searle 1991, 73.

41 Vgl. auch Wingert 2003, 231-240, für eine scharfsinnige Diskussion dieses Arguments.

42 Der Terminus imaginatio ist im Kontext von Spinozas Ethik aus verschiedenen Gründen schwer zu übersetzen. Erstens bezeichnet Spinoza damit - ganz anders als der oft verwendete deutsche Ausdruck „Vorstellungskraft“ kein Vermögen, sondern eine Gattung von bestimmten kognitiven Prozessen; zweitens subsumiert Spinoza nicht nur fiktive Vorstellungen, sondern auch Empfindungen, Emotionen, Zeichenprozesse sowie veridikale Vorgänge wie Wahrnehmungen oder Erinnerungen. Ich werde in der Folge daher den lateinischen Ausdruck verwenden. 
An dieser Stelle ist auf zwei Punkte hinzuweisen. Zum einen ist daran zu erinnern, dass Spinoza zufolge unsere Überzeugungen nicht ausschließlich Produkt der imaginatio sind, sondern dass auch die Möglichkeit zur Bildung von Gemeinbegriffen, notiones communes, besteht. Darunter versteht Spinoza die Begriffe jener nicht-essentiellen, aber notwendigen Eigenschaften, die allen Dingen gemeinsam sind, und deren Kenntnis allein es erlaubt, kausale Interaktionen zwischen den Dingen zu beschreiben. Da sie die allen Dingen gemeinsamen Eigenschaften erfassen, sind die notiones communes - ähnlich wie Descartes' angeborene Ideen - notwendig wahr. Im Unterschied $\mathrm{zu}$ jenen, finden wir sie aber nicht einfach in uns vor, sondern wir müssen sie erst bilden, wozu es unter Umständen gewisser theoretischer Voraussetzungen bedarf. ${ }^{43}$ Für unseren Zusammenhang entscheidender ist, dass es nicht zuletzt die Bildung von notiones communes ist, welche auch die Grundlage für die oben bereits angeführte Unterscheidung zwischen logisch begründeten und psychologisch zufälligen Ideen darstellt. Dank unserer Fähigkeit, solche Begriffe zu bilden, kann also der Wildwuchs der imaginatio in Schranken gehalten werden.

Fast noch wichtiger ist zum anderen, sich zu vergegenwärtigen, dass im Zentrum von Spinozas Konzept der imaginatio ein Prozess der Verarbeitung von körperlichen Affektionen steht. Die imaginatio ist für Spinoza nicht bloß die Produktionsstätte kontingenter Ideenverbindungen, sondern sie stellt, insofern wir es der imaginatio verdanken, dass wir überhaupt Ideen haben, auch eine Quelle jeglichen menschlichen Denkens dar. ${ }^{44}$

43 Andere Deutungen des Begriffs gehen dahin, dass Gemeinbegriffe angeborene Begriffe sind, vgl. dazu jüngst Marshall 2008, 62-66. Es ist mir allerdings nicht klar, wie stark seine Position tatsächlich von meiner entfernt ist. Marshalls Rekonstruktion ist von einer Annahme abhängig, die er explizit nennt, aber nicht diskutiert, nämlich dass Spinoza implizit zwischen bewussten und unbewussten Ideen unterscheidet. Ich teile diese Auffassung. Es stellt sich dann aber die Frage, wie man sich die Bewusstmachung von Ideen, die wir im Prinzip „haben“, zu denken hat. Marshalls Rekonstruktion lässt es - anders als die oben skizzierte - völlig offen, wie man diese Frage angehen könnte. Zu einer detaillierten Erörterung meiner Interpretation vgl. Renz 2010, 278-289.

44 Einer der anonymen Gutachter hat hier eingewendet, dass Gottes Ideen sehr wohl einen kognitiven Gehalt hätten, aber Gott keine imaginatio habe. An dieser Stelle liegt m. E. eine grundlegende Meinungsdifferenz bezüglich der Funktion der Rede von Gottes Ideen in der Ethik vor. M. E. geht Spinoza implizit davon aus, dass eine Idee nur dann aktualiter gehabt werden kann, wenn es mindestens ein endliches Subjekt gibt, das diese Idee hat. Die Rede, dass es in Gott eine Idee von $\mathrm{x}$ gebe, verstehe ich daher so, dass $\mathrm{x}$ im Prinzip vollständig gewusst werden kann und nicht als Aussage über einen tatsächlich vorliegenden kognitiven Akt. Vor dem Hintergrund dieser Auffassung, die ich andernorts ausführlich begründet habe (vgl. Renz 2009b, 429 und insbesondere Renz 2010, 118-124), wären auch zahlreiche Einwände der Gutachter zu erörtern. Aus Platzgründen möchte ich hier nur auf einen Punkt hinweisen. Vor dem Hintergrund der 
Hinzu kommt ein weiterer Punkt, der für die Frage der Wahrheitssensitivität unseres Überzeugungssystems von entscheidender Bedeutung ist. Wie aus 2p16 hervorgeht, versorgen uns imaginäre Ideen auch mit einem kognitiven Gehalt, der in seinen Grundzügen zweifelsohne richtig ist: Wir wissen dank der imaginatio sowohl von uns, von der Existenz anderer uns affizierender Dinge als auch davon, dass irgendwann eine Affektion stattgefunden haben muss. Trotz ihrer ungezügelten Produktivität ist die imaginatio somit eine notwendige Voraussetzung von Erkenntnis in einem starken Sinne des Wortes, denn sie allein macht es möglich, dass endliche Subjekte einen kognitiven Bezug zur Realität haben können.

Paradoxerweise ist es also nach Spinoza ein und derselbe Prozess, der sowohl unser Wunschdenken antreibt, als auch die grundlegende Geist-auf-WeltAusrichtung unseres Denkens stiftet. Der Forderung nach einer Sicherstellung der Wahrheitssensitivität unseres Denkens ist damit allerdings nur bedingt Rechnung getragen. Denn die epistemische Doppelfunktionalität der imaginatio ist dem imaginierenden Subjekt selber meist nicht bewusst. Daher hat, wer sich ihr überlässt, zwar unbezweifelbar einen kognitiven Bezug zur Realität, doch worin genau dieser besteht, ist ihm meist nicht klar. Und auch wenn die imaginatio uns im Prinzip zweifelsfrei anzeigt, dass es außer uns etwas gibt, was uns affiziert, und daher nicht alles Illusion sein kann, so kann es immer noch sein, dass wir uns über die Zeit, den Ort, die Art und die Intensität einer Affektion gehörig täuschen. Dazu kommt, dass die imaginatio uns viel Anlass für allfällige Spekulationen über uns täuschende Götter oder Dämonen gibt, so dass wir selbst der basalen Geist-auf-Welt-Ausrichtung, die sie uns verschafft, verlustig gehen können. ${ }^{45}$ Dieser Gefahr kann nur dann angemessen begegnet werden, wenn wir die Ideen, die der imaginatio entspringen, reflektieren und unter Rückgriff auf Gemeinbegriffe analysieren. Dann können wir uns sowohl vom Wildwuchs zufälliger Ideen befreien, als auch sehen, inwiefern unsere Ideen die Realität angemessen repräsentieren.

skizzierten Meinungsdifferenz lässt sich auch angeben, warum ich Spinozas Auffassung über die Wahrheitssensitivität von Überzeugungen nicht, wie von einem Gutachter vorgeschlagen, am Parallelismus festmache. Der Parallelismus stellt zwar aus der Perspektive Gottes sicher, dass Ideen einen Realitätsbezug haben, er schließt aber nicht aus, dass sich endliche Subjekte darüber irren. Daher kann der Parallelismus zwar eine metaphysische Garantie für die Wahrheit göttlicher Ideen abgeben, er hält aber eo ipso keine Antwort auf die Frage nach Wahrheitssensitivität menschlicher Ideen bereit. Das schließt natürlich nicht aus, dass die Einsicht in den Parallelismus qua notiones communes etwas zur Beantwortung dieser Frage beiträgt. Doch dies tut sie, wie im Haupttext erörtert, zusammen mit der imaginatio.

45 Siehe dazu insbesondere die Aussage, die Spinoza im Tractatus de intellectus emendatione über die Skeptiker macht. Solche Leute seien ihrer selbst nicht inne, oder wie es im Lateinischen heißt: Nam neque seipsos sentiunt. TIE, §47, 38. 
Zusammenfassend können wir somit festhalten, dass Spinozas Ethik der Überzeugungen durch ein komplexes Wechselspiel von imaginatio und notiones communes bestimmt ist, wodurch auch die Wahrheitssensitivität unserer Überzeugungen gesichert wird. So garantiert die Beschaffenheit der imaginatio, in welcher letztlich die Produktion von Ideen wurzelt, dass wir in einem epistemischen Kontakt mit der Realität sind. Dass wir dennoch nicht dem Wunschdenken verfallen, dafür sorgt im Gegenzug die rationale Überprüfung von imaginativ erworbenen Ideen. Im Unterschied zu Descartes' Ansatz wird somit die Wahrheitssensitivität unserer Überzeugungen nicht durch die Einsicht in die Notwendigkeit der Idee Gottes gesichert, sondern dadurch, dass wir die sinnliche Verarbeitung unserer körperlichen Affektionen mit den richtigen begrifflichen Mitteln reflektieren. ${ }^{46}$

AT Adam, C./Tannery, P. (Hg.): Descartes, R. 1988: Euvres. Nouvelle présentation. 12 Bde. Paris.

ST Thomas von Aquin 1934 ff.: Summa Theologica. Deutsch-lateinische Ausgabe. Übers. und komment. von Dominikanern und Benediktinern Deutschlands und Österreichs. Graz u.a.

Allison, H. E. 1987. Benedict de Spinoza: An Introduction. Revised Edition. New Haven/London. Alston, W. P. 1988. „The Deontological Conception of Epistemic Justification“. Philosophical Perspectives 2, 257-299.

Clifford, W. K. 1999. „The Ethics of Belief“. In The Ethics of Belief and Other Essays.

Hg. W. K. Clifford. Amherst, NY, 70-97.

Cottingham, J. 1988. „The Intellect, the Will, and the Passions: Spinoza's Critique of Descartes“. Journal of the History of Philosophy 26, 239-257.

Curley, E. 1975. „Descartes, Spinoza, and the Ethics of Belief“. In Spinoza: Critical Essays.

Hg. E. Freeman/M. Mandelbaum. La Salle, 159-189.

Curley, E./Walsky, G. 1999. „Spinoza's Necessitarismus Reconsidered“. In New Essays on the

Rationalists. Hg. R. J. Gennaro/Ch. Huenemann. Oxford u. a., 241-262.

Davidson, D. 2001. „Actions, Reasons, and Causes“. In Essays on Actions and Events.

Hg. D. Davidson. Oxford, 3-20.

Delahunty, R. J. 1985. Spinoza. London u. a.

Deleuze, G. 1968. Spinoza et le problème de l'expression. Paris.

46 Ich möchte mich bei den anonymen Gutachtern für Ihre äußerst genaue und kritische Lektüre bedanken. Ferner danke ich den Zuhörern und Zuhörerinnen zweier Vorträge, die ich 2010 in Berlin und Graz gehalten habe. Ein großer Dank geht auch an Harald Berger für seinen Kommentar zum Grazer Vortrag. 
Della Rocca, M. 1996. Representation and the Mind-Body Problem in Spinoza. Oxford.

-. 2003. „The Power of an Idea: Spinoza’s Critique of Pure Will“. Noûs 37, 200-231.

Garrett, A. V. 2003. Meaning in Spinoza's Method. Cambridge.

Garrett, D. „Spinoza’s Necessitarianism“. In Yovel (Hg.) 1991, 191-218.

Goldman, A. 1978. „Epistemics: The Regulative Theory of Cognition“. The Journal of Philosophy 75, 509-523.

Greco, J./Turri, J. 2011. „Virtue Epistemology“. The Stanford Encyclopedia of Philosophy.

Hg. E. N. Zalta, URL = <http://plato.stanford.edu/archives/win2011/entries/

epistemology-virtue/>.

Haag, J. 2006. „Descartes über Willen und Willensfreiheit“. Zeitschrift für philosophische Forschung 60, 483-503.

Halbach, V. 2002. „War Descartes erkenntnistheoretischer Voluntarist?“ Zeitschrift für philosophische Forschung 56, 545-562.

Hampe, M. 2004. „Baruch de Spinoza - Rationale Selbstbefreiung“. In Klassiker der Philosophie heute. Hg. A. Beckermann/D. Perler. Stuttgart, 230-250.

Harman, G. 1970. „Knowledge, Reasons, and Causes“. Journal of Philosophy 67, 841-855.

Haslanger, S. 1999. „What Knowledge Is and What It Ought to Be: Feminist Values and Normative Epistemology“. Philosophical Perspectives 19, 459-480.

James, W. 1956. The Will to Believe. Essays in Popular Philosophy. New York/Dover, 1-31.

Kim, J. 2000. „What is 'naturalized epistemology'“?” In Knowledge. Readings in Contemporary Epistemology. Hg. S. Bernecker/F. Dretske. Oxford 2000, 279-297. (Reprinted from Philosophical Perspectives 2, 1988, 381-405.)

Larmore, C. 1984. „Descartes’ Psychologist Theory of Assent“. History of Philosophy Quarterly $1,61-74$.

Loeb, L. 1992. „The Cartesian Circle“. In The Cambridge Companion to Descartes.

Hg. J. Cottingham. Cambridge, 200-235.

Malebranche, N. 1979. Oeuvres. Hg. G. Rodis-Lewis. Paris.

Marshall, E. 2008. „Adequacy and Innateness in Spinoza“. Oxford Studies in Early Modern Philosophy IV, 51-88.

Martin, Ch. 2010. „A New Challenge to the Necessitarian Reading of Spinoza“. Oxford Studies in Early Modern Philosophy V, 25-70.

Nadler, S. 2006. Spinoza's Ethics. An Introduction. Cambridge u. a.

Perler, D. 1998. René Descartes. München.

-. 2006. „Das Problem des Nezessitarismus“. In Baruch de Spinoza: Ethik in geometrischer Ordnung dargestellt. Hg. M. Hampe/R. Schnepf unter Mitarbeit von U. Renz. Berlin, 59-80.

-. $\quad$ 2008. „Begriffliche und psychologische Ordnung bei Spinoza“. Archiv für Geschichte der Philosophie 90, 188-215.

-. 2011. Transformationen der Gefühle. Philosophische Emotionstheorien 1270-1670. Frankfurt.

Prichard, D. 2007. „Recent Work on Epistemic Value“. American Philosophical Quarterly 44, 85-110.

Renz, U. 2003. „Klar, aber nicht deutlich. Descartes’ Schmerzbeispiele vor dem Hintergrund seiner Philosophie“. Studia Philosophica 62, 149-165.

-. 2009a. „Explicable explainers: The Problem of Mental Dispositions in Spinoza’s Ethics.“ In Debating Dispositions. Hg. G. Damschen/R. Schnepf/K. Stüber. Berlin/New York, 79-98. 
-. $\quad 2009 b$ b. „Spinozas Erkenntnistheorie: Eine naturalisierte Epistemologie?“ Deutsche Zeitschrift für Philosophie 57, 419-432.

-. 2010. Die Erklärbarkeit von Erfahrung. Realismus und Subjektivität in Spinozas Theorie des menschlichen Geistes. Frankfurt.

Rooks, M. C. 1995. Philosophical Works and Selected Correspondence of John Locke. Charlottesville.

Rubin, R. 1977. „Descartes’ Validation of Clear and Distinct Apprehension“. Philosophical Review 86, 197-208.

Schütt, H.-P. 1985. „Spinozas Konzept der Modalitäten“. Neue Hefte für Philosophie 24/25, 165-183.

Searle, J. 1991. Intentionalität. Eine Abhandlung zur Philosophie des Geistes. Übers. von H. P. Gavagai. Frankfurt.

Spinoza, B. de 1993. Abhandlung über die Verbesserung des Verstandes. Übers. von W. Bartuschat. Hamburg.

-. 1999. Ethik in geometrischer Ordnung dargestellt. Neu übers., hrsg. und mit einer Einleitung versehen von W. Bartuschat. Hamburg.

Vitz, R. 2010. „Doxastic Voluntarism“. The Internet Encyclopedia of Philosophy. http:// www.iep.utm.edu/doxa-vol/print/, letzte Einsicht 12. 2. 2010.

Williams, B. 1973. „Deciding to Believe“. In Problems of the Self. Hg. B. Williams. Cambridge, 136-151.

-. 1981. Descartes. Das Vorhaben der reinen philosophischen Untersuchung. Aus dem Engl. übers. von W. Dittel/A. Viviani. Weinheim.

Wilson, M. „Spinoza’s Theory of Knowledge“. In The Cambridge Companion to Spinoza. Hg. D. Garrett. Cambridge u. a., 89-141.

Wingert, L. 2003. „Die eigenen Sinne und die fremde Stimme. Über den mehrfachen Grund unserer Wissensansprüche.“ In Wissen zwischen Entdeckung und Konstruktion.

Erkenntnistheoretische Kontroversen. Hg. M. Vogel/L. Wingert. Frankfurt, 218-248.

Wolterstorff, Nicolas 1996. John Locke and the Ethics of Belief. Cambridge.

Yovel, Y. (Hg.) 1991. God and Nature: Spinoza's Metaphysics. Leiden. 